\title{
Accelerator-based facility design in association with nuclear data
}

\author{
H. Takada \\ Japan Atomic Energy Agency, Tokai-mura, Naka-gun, Ibaraki 319-1195, Japan
}

\begin{abstract}
At present, large research infrastructures using high energy and high beam intensity accelerator have been almost realized as examples of SINQ, J-PARC and SNS. In the design study of such accelerator facilities, it is required to seek the best neutronic performance and assure engineering feasibilities such as heat removal from the target and its surroundings, material selection to have enough life-time under radiation environment for reasonable operation duration. It is also important to design sophisticated maintenance scheme including remote handling to treat highly activated components, and optimal radiation shield around them. The roles of nuclear data to these aspects and perspectives are reviewed through the design of J-PARC spallation neutron source.
\end{abstract}

\section{Introduction}

Utilization of accelerator has been spread over a variety of field with the technological progress. As far as the cuttingedge large facilities are concerned, two complementary trends are observed. The first is pursuit of the highest beam energy, which is observed in nuclear and particle physics aiming at the hunt for new particle. Development and upgrade of collider facility such as LEP, RHIC and LHC are typical examples in this category.

The second is the path towards the highest beam power. This performance is desired not only for the nuclear and particle physics but for materials science using synchrotron light sources and neutrons. For neutron science, this movement was triggered by the recommendation of OECD mega science forum for neutron sources that stated the construction of advanced neutron source in three regions: ASIA/Pacific rim, Europe and North America. In Europe, ESS project [1] was proposed to build 5-MW spallation neutron source, but it ended at the design stage. However, SINQ, the steady state neutron source driven by the $590 \mathrm{MeV}$ PSI ring cyclotron succeeded to receive $1 \mathrm{MW}$ beam and seeks for upgrade of the beam power $[2,3]$. USA and Japan started big project independently for construction of 1.4 MW and $1 \mathrm{MW}$ pulsed neutron source, respectively. In 2006, SNS [4] was completed at ORNL and now raising beam power towards full specification in the commissioning process. Japanese spallation source (JSNS) is on the final stage of component installation in the J-PARC project [5] in which Nuclear \& Particle Physics Facility, Nuclear Transmutation Experiment Facility and Neutrino Facility are included.

Nuclear transmutation by ADS (Accelerator Driven system) also needs the high power beam of tens of MW. Conceptual system design activities are in progress inclusively with the experimental studies of sub-critical reactor physics using an external source, and engineering feasibility studies on the handling of liquid metal eutectic $[6,7]$. To meet the request for the accumulation of high quality neutron data on minor actinide and fission products for detailed neutronic study, some nuclear data measurements are under progress in the programs of $\mathrm{n}_{-}$TOF [8], EUROTRANS [9] and Innovative Nuclear Energy System Technology (INEST) Development projects [10]. At JSNS, a neutron beam line aiming at measuring neutron cross sections of minor actinides and fission products is under construction.

Component design based on the neutronics design using nuclear data faces to some issues inherent for the utilization of a high power beam. Engineering feasibility is assured in view of thermal stress and cooling capability of components in which heat deposition is induced by the nuclear heating. Life estimation of structural material due to radiation damage is also important to ensure acceptable operation duration. It is noted that pulsed neutron source is exposed in much severer condition than the steady state one. This paper reviews roles of nuclear data in the accelerator-based facility design through the latest activities of the JSNS design.

\section{Roles of nuclear data}

Design study of the pulsed spallation neutron source requires nuclear data in the broad energy region ranging from $\mathrm{GeV}$ down to sub-meV. Nuclear data contributes to the following design issues: 1) target-moderator-reflector design pursuing the best performance in pulse intensity, narrow pulse width and short tail, 2) reasonable biological shield based on the best choice in materials and dimension of components, 3) assessment of induced radioactivity of components, coolant and ventilation air for design of ancillary system such as casks, off-gas handling systems, cooling water and ventilated air systems, 4) DPA of components for their life estimation, and 5) Nuclear heating to engineering design of components. The issue 1) is inherent to the pulsed neutron source. In particular, comprehension of neutron behaviour at the thermal to cold region is important. The others are common to the design of a high power accelerator, secondary particle production targets and a beam dump. They are in close relation with each other and elemental factors to determine not only the specification of the facility itself but also accelerator components. For example, the beam profile is the initial condition of heat deposition and radiation damage in a target and its vessel. Therefore 
the specifications of magnets and related components are determined to transport beam having acceptable profile to the requirements from the target system.

\section{Data, code and methodology}

Spallation reaction involves many kinds of particles as proton, neutron, pions in broad energy range from incident to low energies. To simulate the behaviour of those particles in the neutronics design of the existing facilities, a high energy particle transport code including intra-nuclear cascade evaporation model (INCE) has been employed commonly in connection with a conventional particle transport codes using the processed data from nuclear data files.

Progress in development of the nuclear data library LA150 [11] and a contemporary code system MCNPX [12] provided more reliable and convenient tool than ever. They were employed in the design of ESS and SNS. The former improved the weakness of INCE in the energy range from 20 to $150 \mathrm{MeV}$ by the evaluation using more reliable physics model. The latter provides a variety of tally as built in the MCNP code series $[13,14]$ to get physical aspects of flux, nuclide yield, heat deposition and so on. Successive updates of MCNPX are going on [15].

At low energy region, scattering kernel is needed to simulate the neutron scattering with the moderator materials of hydrogen, methane and so on. The data derived from MacFarlen [16] is in the available form in MCNP.

The same concept has been introduced in the neutronics design tool of JSNS. The code system and data libraries are shown in figure 1. The PHITS code [17] is a multipurpose particle and heavy ion transport Monte-Carlo code based on NMTC/JAM [18] and MCNP. Evaporation model was upgraded to the generalized evaporation model GEM [19] to improve the production of composite nuclei. LA-150 and JENDL-3 library [20,21] was used in the MCNP part. Flux to dose conversion coefficient extended to $\mathrm{GeV}$ region [22] was also given for dose assessment.

For estimation of the build-up and decay of induced radioactivity in materials, the DCHAIN-SP2001 [23,24] code was employed, where the nuclide yield obtained by PHITS was used as source term. The time evolution of radioactive nuclides is calculated using Bateman equation taking account of the nuclear reaction by neutron field. Activation cross section was taken from FENDL-Dosimetry file [25] with an adjustment [26] based on the experimental data.

For DPA calculation, displacement cross section compiled in LA150 library was used up to $150 \mathrm{MeV}$, while it was obtained with Lindhard-Robinson model $[27,28]$ at energy region higher than $150 \mathrm{MeV}$ using the information of the recoiled nucleus calculated by PHITS [29]. The resultant displacement cross section of ${ }^{56} \mathrm{Fe}$ for neutron incidence is shown in figure 2. A discrete jump between the results of LA150 and PHITS is observed at $150 \mathrm{MeV}$.

\section{Validation of data and code}

Stringent validation of data and code is indispensable to know their accuracy. The following activities provided appreciable

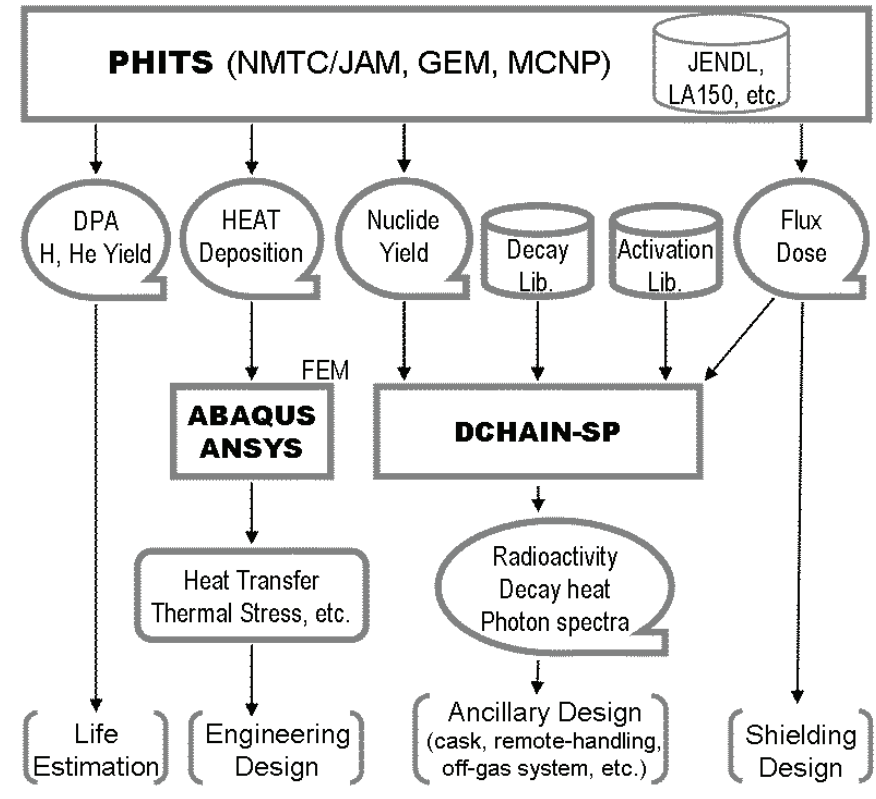

Fig. 1. Data, codes and their roles for design of the spallation neutron source of J-PARC.

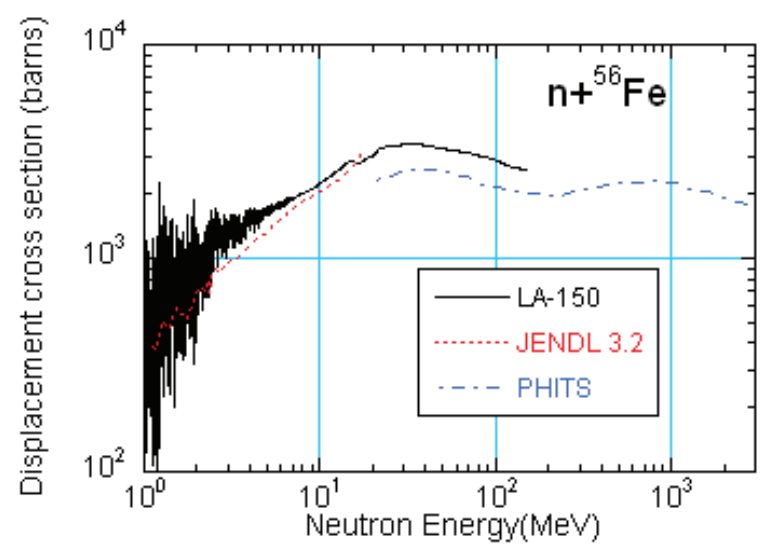

Fig. 2. Displacement cross section of ${ }^{56} \mathrm{Fe}$ for neutron incidence.

data in the intermediate energy region from tens of $\mathrm{MeV}$ to $\mathrm{GeV}$. Spallation experiments using thick and thin targets [30-33], and beam dump [34] at KEK, those with stopping length targets [35] at TIARA ensured the accuracy of intranuclear cascade model for neutron energy spectra. A series of experiments $[36,37]$ on the transmission of quasi monoenergetic neutrons leaded to the improvement of the treatment of the elastic scattering cross section in the code system at tens of $\mathrm{MeV}$. The labyrinth experiment [38] also produced the data for the design of access routes to the accelerator beam tunnel. The energy deposition measurements performed in Russia [39-41] was also useful to know the accuracy of codes.

From the viewpoint of the engineering design of functional components and licensing, data production using realistic mock up is strongly needed. ASTE collaboration [42] dedicated on a mercury target system is worth to be highlighted in this point of view. The following data were measured using 1.6 to $24 \mathrm{GeV}$ protons at AGS: 1) neutron spectral index from the 


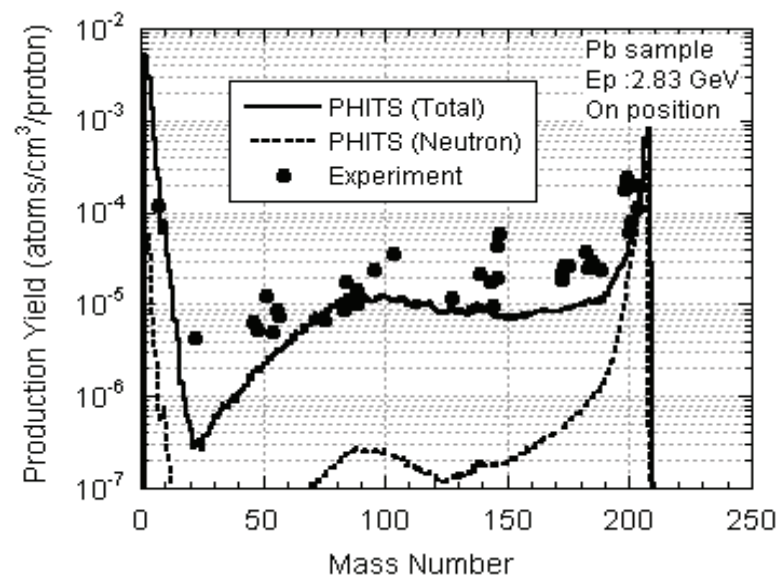

Fig. 3. Nuclide production yield in Pb sample on mercury target for $2.83 \mathrm{GeV}$ proton incidence. Solid mark is measured data. Solid line stands for the result of PHITS calculation in which contribution of secondary neutrons from target is shown by the dotted-line.

target- $\mathrm{H}_{2} \mathrm{O}$ moderator with $\mathrm{Pb}$ reflector system by the currentmode TOF and foil activation techniques, 2) attenuation property of neutrons at lateral shields made of steel or concrete, and 3) spallation products produced in various samples on the target. Time evolution of the products was measured so that dominant nuclides after certain cooling period could be identified. The measured data for $\mathrm{Pb}$ sample is shown in figure 3 [43] with calculated results.

It has been confirmed using the whole data that analyses underestimated the measured data by about a factor of 2 although more significant discrepancy was observed for some nuclides such as intermediate mass fragments and meta-stable ones $[43,44]$. Considering the underestimation of neutron energy spectra at tens of $\mathrm{MeV}$ regions, the accuracy of the code system has been validated as a factor of 2 [45].

For the energy deposition in target materials, comparison of calculated results with experimental ones is shown in figure 4 [46]. The calculated results are in good agreement with the measured data caused mainly by the slowing down of primary protons in the thick lead target. On the other hand, it was found that the nuclear heating number (kerma factor) based on JENDL-3.2 was improper because the energy balance of the neutron reaction did not hold when any adjustment was done in the evaluation procedure. A re-evaluation was carried out by counting the energy of emitted particles from history data of the MCNP calculation, so that the data was modified as exampled in figure 5 [47].

\section{Effect of nuclear data in design}

In this section, the relationship between the nuclear data and the facility design is discussed using the results of JSNS. In figure 6, a three-dimensional cut-away view of the JSNS target station is depicted. Three supercritical hydrogen moderators are placed in "wing-geometry" with respect to the mercury target surrounded with the inner beryllium and outer steel reflector [48]. The upper two moderators provide superior

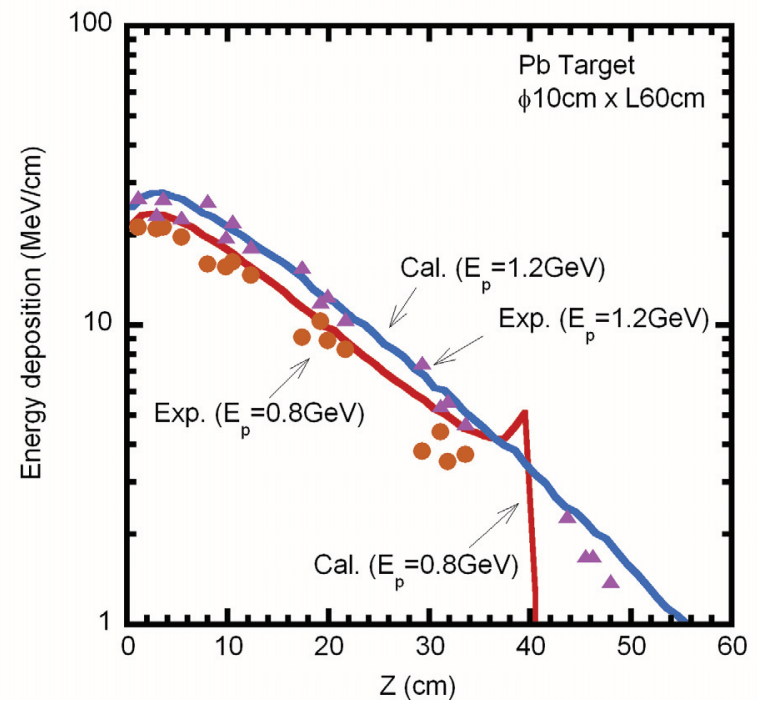

Fig. 4. Energy depositon in thick lead target of $10 \mathrm{~cm}$ diameter and $60 \mathrm{~cm}$ in length. Circles and triangles stand for the measured data for 0.8 and $1.2 \mathrm{GeV}$, respectively. Solid line represents the results calculated with PHITS.

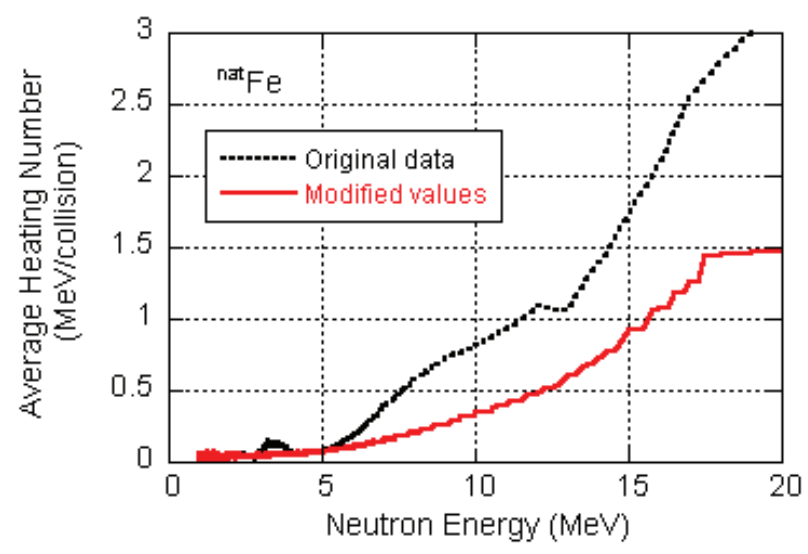

Fig. 5. Nuclear heating number of ${ }^{\text {nat }} \mathrm{Fe}$ for neutron reaction. Dotted and solid lines stand for the data built in the MCNP code and those modified based on Monte Carlo calculation.

pulse characteristics with narrow peak width using Ag-InCd alloy as decoupling (neutron absorption) material $[49,50]$. The lower one is for highest peak and time-averaged intensity by surrounded with $\mathrm{H}_{2} \mathrm{O}$ pre-moderator for enhancement of neutron intensity [51]. $3 \mathrm{GeV}$ protons are injected on the mercury target with a profile of $18 \times 7 \mathrm{~cm}^{2}$ footprint in uniform plus Gaussian configuration in phase space [52].

\subsection{Neutronic performance of moderator}

Hydrogen exists in two forms of ortho and para- $\mathrm{H}_{2}$. Total cross sections for both forms are shown in figure 7 [53] with experimental data. Moderation of neutron is promoted in the para-hydrogen rather than ortho- by means of the para to ortho spin-flip transition between the two states. Lower cross section of para-hydrogen suggests that $100 \%$ para- $\mathrm{H}_{2}$ with a large volume moderator could provide the best neutronic 


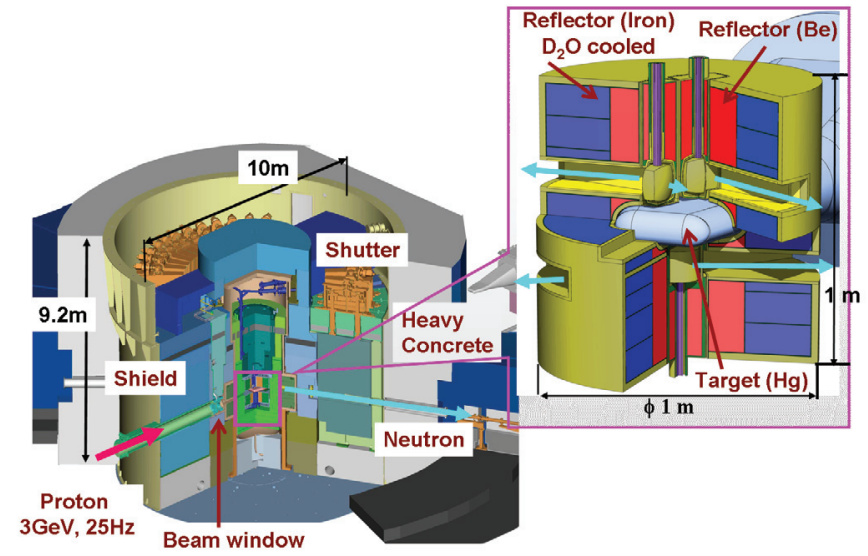

Fig. 6. View of the target station of JSNS. The target $(\mathrm{Hg})-$ moderator-reflector portion is enlarged.

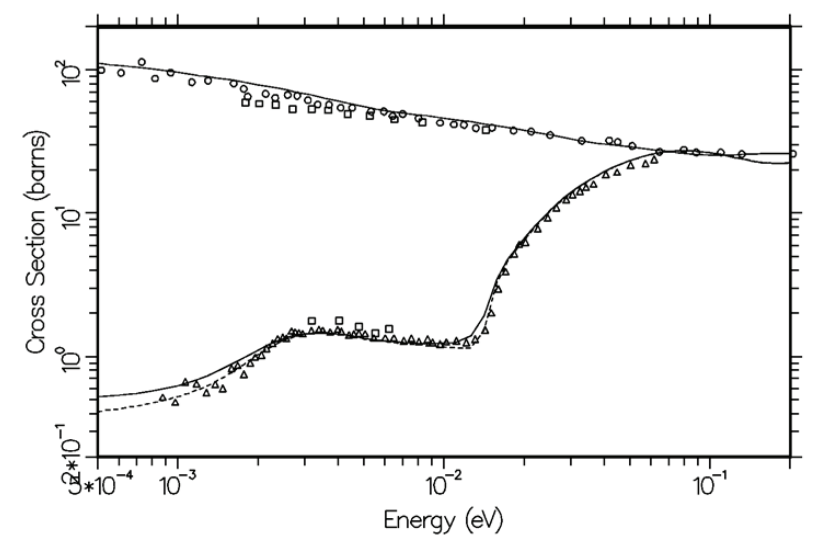

Fig. 7. Calculated values of total scattering cross sections for liquid ortho-hydrogen (upper curve) and liquid para-hydrogen (lower curve) are compared with experimental data. Solid and dashed cueves are at $20 \mathrm{~K}$ and $14 \mathrm{~K}$, respectively.

performance. Experimental studies proved the enhancement of neutron intensity using higher fraction of para- $\mathrm{H}_{2}$ [54].

In figure 8 , the estimated pulse intensity of $2 \mathrm{meV}$ neutron at emission surface of a moderator of JSNS is shown with that of SNS. Peak intensity in J-PARC is expected to be about 4.5 times higher than SNS. Considering that J-PARC inherently has a gain factor of 2.4 against SNS equal to the repetition rate ration between $25 \mathrm{~Hz}$ and $60 \mathrm{~Hz}$, the effective gain is 1.8. This comes from the following different design concepts between the facilities: 1) another moderator with neutron absorber is allocated just next to the moderator at SNS, 2) difference of moderator size. This means careful design of the targetmoderator geometry and the choice of the ortho-para ratio lead to large difference on the neutron performance.

As for the data for hydrogen in the cold neutron region, the scattering data is scare. In addition, finite ambiguity is observed around $20 \mathrm{meV}$ [55] though experiments. It is of interest to measure scattering data at this region for high performance cold moderator system and evaluation of some proposed scattering kernels [56,57].

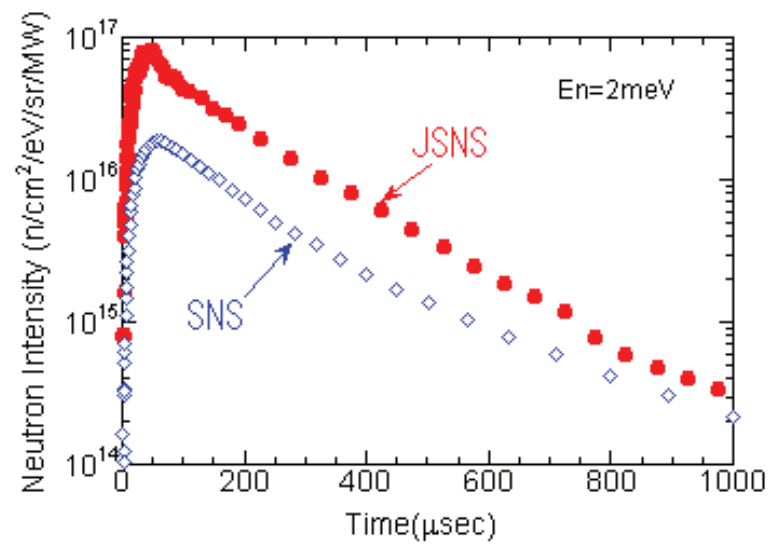

Fig. 8. Pulse intensity of $2 \mathrm{meV}$ neutron as a function of emission time at viewed surface of liquid hydrogen coupled moderator. Calculated results of JSNS and SNS [58] systems are represented by solid and open marks, respectively.

\subsection{Induced radioactivity}

Spallation products have broad mass distribution from target to light nuclei as ${ }^{7} \mathrm{Be}$. The induced radioactivity is the base for making a maintenance scenario. Moreover, the estimation of ${ }^{3} \mathrm{H}$ and noble gas are important in view of radiation safety. In JSNS, mercury target with vessel and moderator-reflector assembly are highly activated. In order to handle these components at maintenance, the induced activity has been calculated under the condition that the operation time is $5000 \mathrm{~h}$ in a year. The amount of radioactivity in the components of the mercury target and its container, the moderator chamber and the reflector were estimated [59] at their life time to be the order of $10^{4} \mathrm{TBq}(30 \mathrm{y}), 10^{3} \mathrm{TBq}(0.5 \mathrm{y}), 10^{2} \mathrm{TBq}(6 \mathrm{y})$ and $10^{3} \mathrm{TBq}(6 \mathrm{y})$, respectively, where the number in parenthesis indicates the lifetime of individual component.

A transfer cask accommodating the moderator-reflector assembly with a steel plug has been designed [60] based on the inventory in it. The dimension of the assembly with the plug is $1.5 \mathrm{~m}$ in maximum diameter and $3.65 \mathrm{~m}$ in height. Resultant dimension of the steel cask was estimated as $1.9 \mathrm{~m}$ inner diameter with $29 \mathrm{~cm}$ shield thickness at maximum. This caused the weight of the cask to be about 120 tons including the assembly, so that a $130 \mathrm{t}$ ceiling crane was installed in the facility to handle it. The similar design procedure was taken to the other components of the ancillary system. Moreover, an off gas system is under design to handle ${ }^{3} \mathrm{H}$ and noble gas of about $100 \mathrm{TBq}$ in the estimated value.

\subsection{DPA in components}

DPAs is the measure to evaluate the life time of components and this is the key factor to determine the facility's operation performance including the cost required maintenance work. The major components exposed to high radiation field were calculated under the condition that the operation time in a year is $5000 \mathrm{~h}$ at beam power of $1 \mathrm{MW}$. The results are shown in table 1 [29] with allowable values, in which the contribution of proton is also listed. As far as the mercury target vessel is 
Table 1. Comparison of DPA in major components in JSNS.

\begin{tabular}{lllll}
\hline Component & Material & \multicolumn{2}{l}{ DPA/5000MWh } & Allowable \\
Vessel & & Total & Proton & Value \\
\hline Target & 316L-SS & 3.9 & 0.81 & 5 \\
Reflector & A5083 & 2.8 & 0.02 & 20 \\
Moderator & A6061-T6 & 2.8 & 0.02 & 20 \\
Beam window & A5083 & 0.44 & 0.33 & 10 \\
\hline
\end{tabular}

concerned, the pitting damage was more severe condition to determine its lifetime.

As shown in figure 2, a significant difference is observed between the displacement cross section obtained with LA-150 library and that calculated by PHITS. The latter is about $20 \%$ lower and this trend seems to continue even at higher energy region. Further study is needed to consistency between the LA-150 based data and the INCE based ones. However, the fraction of the neutrons above $100 \mathrm{MeV}$ is extremely low for the target-moderator-reflector assembly. The ambiguity in the displacement cross section in high energy region affects only about several percent on DPA at most.

\subsection{Nuclear heating in components}

Nuclear heating is the initial condition of the engineering design of thermal stress, heat removal and so on. The nuclear heating in some components calculated with the modified heating number is listed in the second column of table 2 [47]. For comparison, the calculated results with the kerma data obtained by JENDL-3 are summarized in the third column. No significant difference is observed between the two results because of the small fraction of neutrons having energies from 1 to $20 \mathrm{MeV}$. However, the modification of the heating number shown in figure 5 suggests that energetically correct treatment of nuclear reaction is required for precise estimation. This is true for secondary neutrons in all energy range. Since the energy balances for the elastic, discrete inelastic and capture reactions are treated correctly in the data library, it is of interest to investigate this effect for other reactions such as $(n, p),(n, x)$. On the other hand, the number and energy of the produced secondary neutron is the substance as initial conditions. It is needed to evaluate the energy spectra of particles emitted via nuclear reactions precisely.

The same approach was applied to the energy loss of electrons accompanied with the photon transport $[46,47]$. Since kerma factor for photon is given as an one-point approximation in the code, it has possibility to overestimate the heating by photons having energies to path through a defined portion, and underestimate the heating by the secondary photons created in that portion because their information is not included in the kerma factor. For comparison, the calculated results using the built-in photon kerma factor is listed in the column 4 of table 2 . It is observed that this modification improved accuracy of the nuclear heating about $20 \%$ in the JSNS design.

\section{Concluding remarks}

Various design issues related to nuclear data have been studied in close connection with the engineering design, the material
Table 2. Estimated nuclear heating in target-moderator-reflector components at $1 \mathrm{MW}$ operation in JSNS. Case-1 is the result using heating number based on JENDL 3 for neutron. Case-2 is that for photon.

\begin{tabular}{llll}
\hline Component & \multicolumn{3}{l}{ Heat deposition $(\mathrm{kW})$} \\
& Designed & Case-1 & Case-2 \\
\hline Hg target & 450.5 & 452.3 & 478.7 \\
Target Vessel & 42.5 & 42.4 & 38.3 \\
Reflector with vessel & 193.9 & 195.3 & 183.9 \\
Moderator (H2) & 0.76 & 0.76 & 0.68 \\
Moderator vessel & 0.63 & 0.61 & 0.54 \\
\hline
\end{tabular}

damage estimation and the radiation safety for construction of the 1-MW pulsed spallation neutron source. The following roles and perspectives of nuclear data have been derived through the design studies.

1) Stringent benchmarking using realistic mock up is important to validate the design tools.

2) The induced activity is important as the initial condition to determine the specifications of the ancillary system and maintenance scenario. Further data production and code development is needed in relation with realistic model in broad energy range and the nuclides involved. It is of interest to investigate the effect of the latest improvement $[61,62]$ of INCE model on the design items.

3) Material strength is the key factor to determine the facility's operation performance at the high power accelerator facility. Closer relation with materials research would be needed to study the correlation between nuclear data such as DPA, $\mathrm{H}$ and $\mathrm{He}$ accumulation and the materials damage property. The results of MEGAPIE project [63] would be useful for further studies.

4) Heat deposition is also important issue in view of engineering design. For the high power accelerator facility, it is necessary to estimate the contribution of secondary particle accurately. The data development in this viewpoint is required.

The presented design items would give useful information not only to the sophisticated design and R\&D for the applications using high power beams such as ADS and heavy ion facilities but also the further compilation of the high energy nuclear files such as JENDL-HEF [64]. Finally, J-PARC itself would be one of tools to produce nuclear data through operation for further development of accelerator applications.

The author would like to thank to Drs F. Maekawa, M. Teshigawara, M. Harada and T. Kai, the member of the neutronics design team of JSNS, for their useful comments on this article.

\section{References}

1. K.N. Clausen, in Proceedings of ICANS-XVI The 16th Meeting of the International Collaboration on Advanced Neutron Sources, ESS 03-136-M1 (2003), p. 61.

2. W. Wagner, in Proceedings of ICANS-XVI The 16th Meeting of the International Collaboration on Advanced Neutron Sources, ESS 03-136-M1 (2003), p. 53. 
3. W. Wagner, in Proceedings of ICANS-XVII The 17th Meeting of the International Collaboration on Advanced Neutron Sources, LA-UR-06-3904 (2006), p. 102.

4. Spallation Neuron Source Project Completion Report, SNS 100000000-BL-0005-R00 (2006).

5. S. Nagamiya, J. Nucl. Sci. Technol. Suppl. 1, 40 (2000).

6. H. Oigawa et al., in Proceedings of International Conference GLOBAL 2005 Nuclear Energy Systems for Future Generation and Global Sustainability (AESJ 2005, CDROM), p. 272.

7. J.N Knebel et al., Actinides and fission product partitioning and transmutation (OECD NEA, 2005, 157), p. 467.

8. A. Mengoni et al. (eds.), CERN-INTC-2005-021 (2005).

9. F. Goldenbaum (these proceedings.)

10. M. Igashira et al., in Proceedings of Workshop on nuclear data for the transmutation of nuclear waste: nuclear science and technology in the service of mankind, edited by A. Kelic, H.K. Schmidt (Gesellschaft fuer Schwerionenforschung mbH, 2003, [CD-ROM]), p. 7.

11. M.B. Chadwick et al., Nucl. Sci Eng. 131, 293 (1999).

12. L.S. Waters (ed.), LA-UR-99-1995 (1999).

13. J.F. Briesmeister (ed.), LA-12625-M (1997).

14. J.F. Briesmeister (ed.), LA-13709-M (2000).

15. D.B. Pelowitz et al., in Proceedings of ICANS-XVII The 17th Meeting of the International Collaboration on Advanced Neutron Sources, LA-UR-06-3904 (2006), p. 628.

16. R.E MacFarlane, LA-12639-MS (1994), R.E. MacFarlane, D.W. Muir, LA-12740-M (1994).

17. H. Iwase et al., J. Nucl. Sci. Technol. 34, 1142 (2002).

18. K. Niita et al., Nucl. Instrum. Meth. Phys. Res. B 184, 406 (2001).

19. S. Furihata, Nucl. Instrum. Meth. Phys. Res. B 171, 251 (2000).

20. T. Nakagawa et al., J. Nucl. Sci. Technol. 32, 1259 (1995).

21. K. Shibata et al., J. Nucl. Sci. Technol. 34, 1171 (1997).

22. Y. Sakamoto et al., JAERI-Tech 2001-042 (2001) (in Japanese).

23. H. Takada et al., JAERI-Data/Code 1999-008 (1999).

24. T. Kai et al., JAERI-Data/Code 2001-016 (2001) (in Japanese).

25. A.B. Pashchenko, INDC(NDC)-341 (IAEA) (1996).

26. T. Kai et al., in Proceedings of ICANS-XVI The 16th Meeting of the International Collaboration on Advanced Neutron Sources ESS 03-136-M1 (2003), p. 141.

27. M.J. Norgett et al., Nucl. Eng. Des. 33, 50 (1974).

28. J. Lindhard et al., Mat. Fys. Medd. Dan. Vid. Selsk. 36, 10 (1963).

29. M. Harada et al., J. Nucl. Mater. 343, 197 (2005).

30. S. Meigo et al., Nucl. Instrum. Meth. Phys. Res. A 431, 521 (1999).

31. S. Meigo et al., J. Nucl. Sci. Technol. Suppl. 2, 1252 (2002).

32. S. Meigo et al., International Conference on Nuclear Data for Science and Technology, edited by R.G. Haight, M.B. Chadwick, T. Kawano, P. Talou (AIP Conference Proceedings 769, 2005), p. 1513.

33. K. Ishibashi et al., J. Nucl. Sci. Technol. 34, 529 (1997).
34. H. Takada et al., Nucl. Sci. Eng. 135, 23 (2000).

35. S. Meigo et al., International Conference on Nuclear Data for Science and Technology, edited by G. Reffo, A. Ventura, C. Grandi (Italian Physical Society, Conference Proceedings 59 PART1, 1997), p. 413.

36. N. Nakao et al., Nucl. Sci. Eng. 124, 224 (1996).

37. H. Nakashima et al., Nucl. Sci. Eng. 124, 243 (1996).

38. Su. Tanaka, et al., Health Phys. 81, 406 (2001).

39. V.I. Belyakov-Bodin et al., Nucl. Instrum. Meth. Phys. Res. A 295, 140 (1990).

40. V.I. Belyakov-Bodin et al., Nucl. Instrum. Meth. Phys. Res. A 314, 508 (1992).

41. V.I. Belyakov-Bodin, et al., Nucl. Instrum. Meth. Phys. Res. A 335, 30 (1993).

42. H. Nakashima et al., J. Nucl. Sci. Technol. Suppl. 2, 1155 (2002).

43. Y. Kasugai et al., in Proceedings of the Symposium on Nuclear Data, JAEA-Conf 2006-009 (2006), p. 136.

44. T. Kai et al., in Proceedings of the Symposium on Nuclear Data, JAEA-Conf 2006-009 (2006) 130.

45. H. Nakashima et al., Raidat. Prot. Dos. 115, 564 (2005).

46. F. Maekawa (private communication).

47. M. Harada et al., The 18th Meeting of the International Collaboration on Advanced Neutron Sources (2007) (submitted)

48. M. Teshigawara et al., in Proceedings of ICANS-XVI The 16th Meeting of the International Collaboration on Advanced Neutron Sources, ESS 03-136-M1 (2003), p. 601.

49. M. Harada et al., Nucl. Instrum. Meth. Phys. Res. A 539, 345 (2005).

50. M. Harada et al., Nucl. Instrum. Meth. Phys. Res. A (in press).

51. T. Kai et al., Nucl. Instrum. Meth. Phys. Res. A 523, 398 (2004).

52. S. Meigo et al., Nucl. Instrum. Meth. Phys. Res. A 562, 569 (2006).

53. R.E MacFarlane, in Proceedings of International Workshop on Cold Moderators for Pulsed Neutron Sources (Paris, OECD 1997), p. 221.

54. M. Ooi et al., in Proceedings of ICANS-XVI The 16th Meeting of the International Collaboration on Advanced Neutron Sources, ESS 03-136-M1 (2003), p. 791.

55. M. Ooi, Thesis, Hokkaido University, 2004.

56. N. Morishima et al., Nucl. Instrum. Meth. Phys. Res. A 350, 275 (1994).

57. J.R. Granada et al., Physica B 348, 6 (2004).

58. http://www.sns.gov/instrument_systems/components/HPTSsct.shtml

59. T. Kai et al., J. Nucl. Sci. Technol. Suppl. 4, 172 (2004).

60. M. Teshigawara et al., JAERI-Tech 2005-029 (2005).

61. A. Bouldard et al, Phys. Rev. C 66, 044615 (2002)

62. T. Aoust et al., Nucl. Instrum. Meth. Phys. Res. A 562, 810 (2006).

63. G.S. Bauer et al., J. Nucl. Mater. 296, 17 (2001).

64. Y. Watanabe et al., International Conference on Nuclear Data for Science and Technology, edited by R.G. Haight, M.B. Chadwick, T. Kawano, P. Talou (AIP Conference Proceedings 769, 2005), p. 326. 